\title{
Observing Convection in Microgravity
}

\author{
Matt Heer \\ East Troy High School Physics
}

\begin{abstract}
.
The purpose of the experiment is to observe the movement, or lack thereof, of heat in an enclosed space in multiple gravitational accelerations. An electric heat source will be placed in the center of an insulated box and three temperature probes above and below the heating element at equal spacing will record temperature variations every hundredth of a second.

It will be placed on a $\mathrm{KC}-135$ airplane that does a series of parabolas that provide an acceleration of $0 \mathrm{G}$ (or microgravity) similar to that of astronauts on the space station, and $2 \mathrm{G}$ on its return climb.
\end{abstract}

On earth heat is dispersed unevenly due to cold air being denser therefore getting pulled down by gravity. In a micro gravitational environment there is nothing to cause this shift. During the microgravity phase of our experiment, we expect to initially see symmetry in the temperatures (ie temperature probe $\mathrm{C}$ and $\mathrm{D}$ are the same, $\mathrm{B}$ and $\mathrm{E}$ are the same, $\mathrm{A}$ and $\mathrm{F}$ are the same. See Illustration 1). As the plane goes into its $2 \mathrm{G}$ phase, we expect to see heat rise. (ie probe $\mathrm{A}$ is hottest, $\mathrm{B}$ next hottest, and continuing with that pattern with probe $\mathrm{F}$ being coldest)

\section{Background}

This experiment is testing the dispersal of heat in different levels of gravity. In order to understand this, the theory of convection needs to be understood. Convection is when the less dense hot air stagnates above the dense cold air. Convection relies on gravity because it separates the air

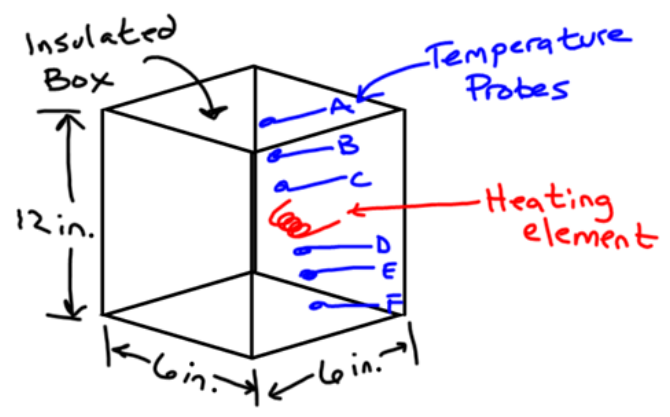
based on density.

Another method of heat is radiation. With radiation, heat energy is directly transported through space by electromagnetic waves. This type of heating, unlike convection, is not affected by gravity. That's why heat from the sun can be felt on Earth. It would be possible, in a vacuum, to test the effects that heat via radiation would have on our readings from the temperature probes. However, we don't have the means at this time. It's our hypothesis, though, that the effect will be infinitesimal.

\section{Equipment Design}

Overview. Our heat dispersal experiment is designed with simplicity, effectiveness, and space efficiency in mind. There will be a metal frame surrounding our main insulating box that will strap to the bottom of the plane. The main insulating box will be attached to the frame and self-contained. All internal components of the box will be secured and no hazardous materials will be used. There will be a variac to control the amount of current flowing to the nichrome heating element. There will be a switch to turn on/off the flow of current from the power source 
to the variac which goes directly to the heating element. It will be fused at 10 amps as a backup fail-safe. A laptop will be strapped to the top to take in data from our data acquisition unit (DAQ). The DAQ will have 6 thermocouples attached to it leading into the insulated box that will take temperature data. It will be logged in the computer for later analysis.

Heating Element Set-Up. The heat source will be set up in the very center of the box. It will be a piece of nichrome wire (commonly found in toasters and electric stoves) wound in a tight spiral that is controlled by a variac and fused at a maximum of 10 amps. See illustration 2 below for a view of how this is set up inside the box.

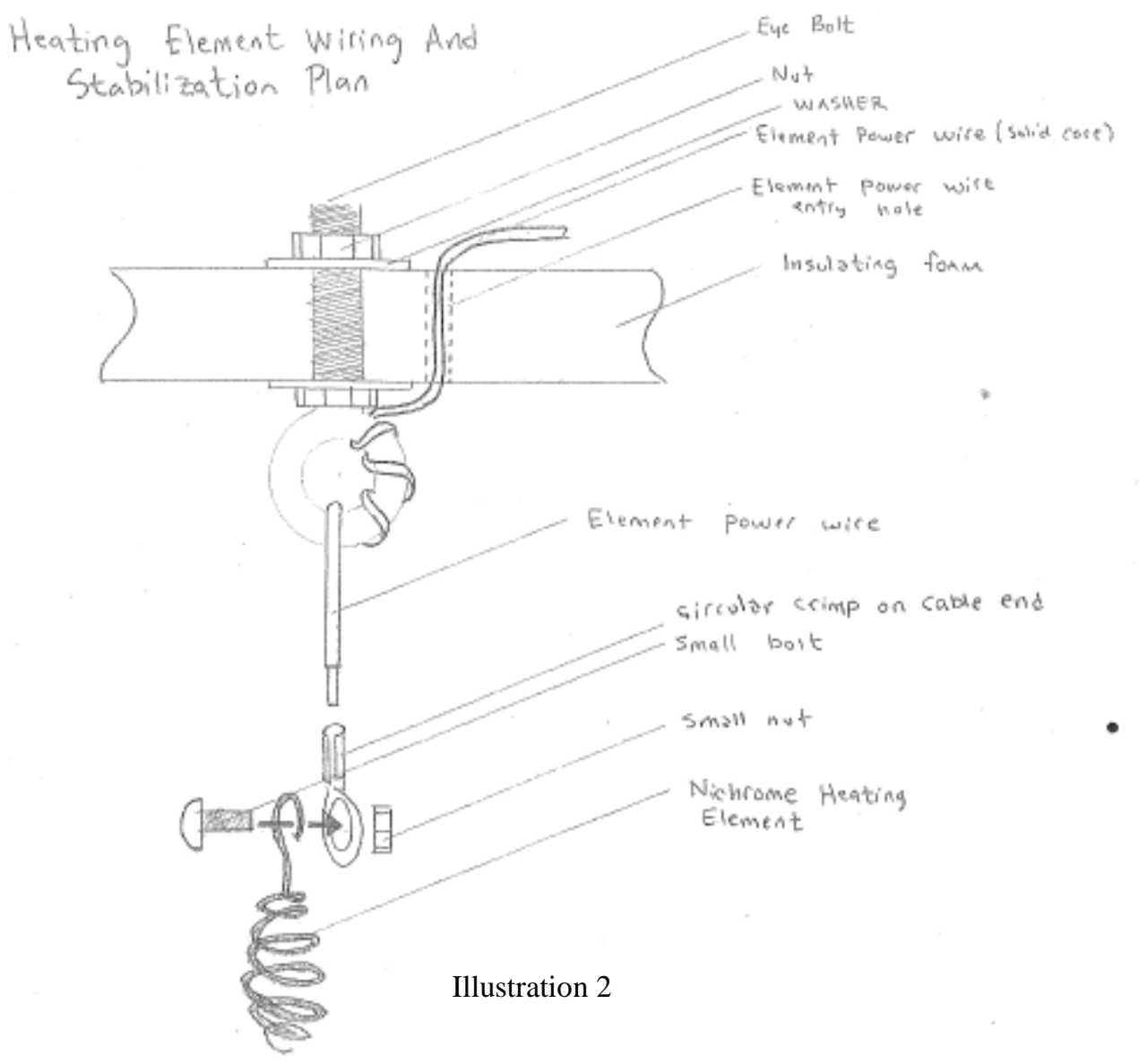

Main Insulating Box. The main box for our experiment will be made of a rigid insulating foam material. The outer dimensions of it will be approximately 20 " $\mathrm{x} 20$ "x 20 ". The inner dimensions will be 6"x6"x12" with our electronic equipment on its surface. The material we are using is residential construction insulating foam commonly used in between the studs of a house.

Stabilizing Frame. The stabilizing frame will be made out of aluminum L brackets. It will be secured together with 3/8" diameter grade 5 nuts and bolts. It would be secured via ratcheting straps to the floor of the plane. Any edges of the frame that are showing will be covered with pipe insulating foam to protect occupants of the plane from cutting themselves during the weightless phases. See Illustration 3 on the next page. 


\section{Exterior Foll View}

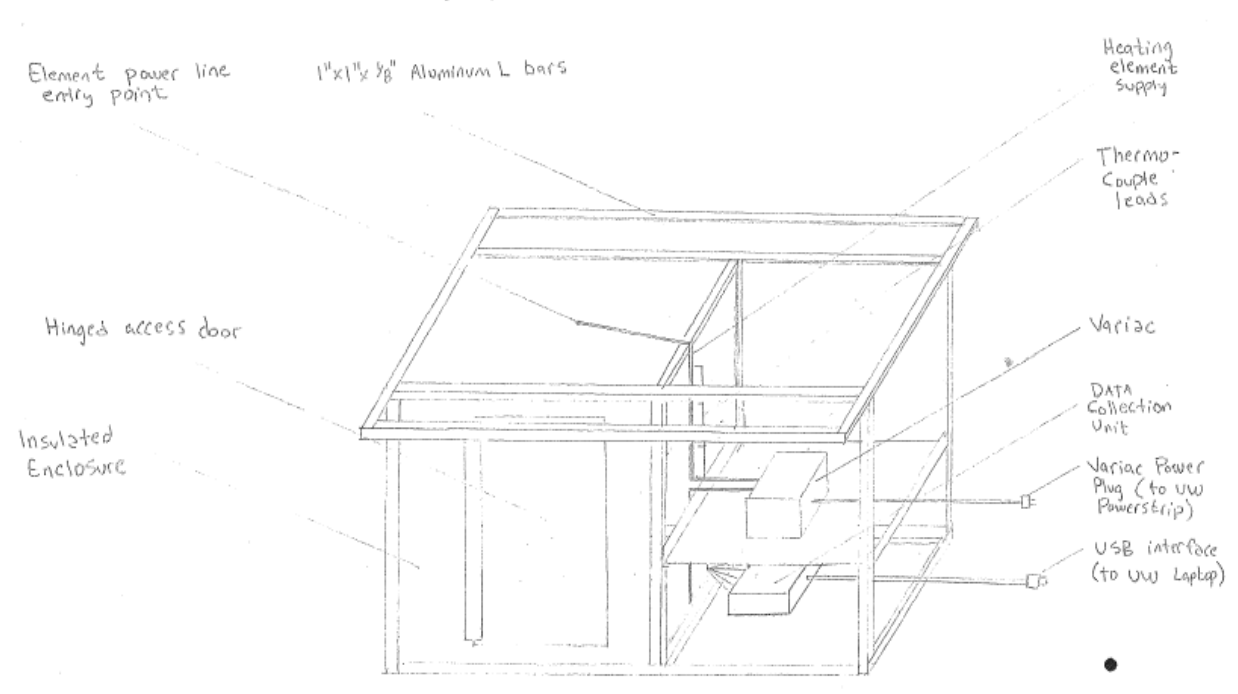

Illustration 3

Temperature Probes. Distributed evenly from the heating source every 2 inches from the center of the box, we will have temperature probes. They will be attached to data logger on the outside of the box, and go through the material to the box's interior to collect data. To keep them stabilized, stainless steel TIG welding wire will be taped to them so they will not move during the $2 \mathrm{G}$ phase of the flight or if there are any unexpected bumps or turbulence.

Electronics. The data logger will record the change in temperature over time and in relation to distance from each probe. They will run off of batteries for the duration of the flight and be attached to a laptop which will be strapped to the top of the frame. The computer will also run off its own internal battery.

\section{Procedure}

The experiment was run by a laptop which was velcroed and strapped to the top of the frame. A data acquisition device (DAQ) was connected via USB cable to the laptop. 6 thermocouples were run into the enclosed insulated box and they were programmed to take 100 measurements per second. The heating element was switched on when the plane is in its 'Zero G' phase, accelerating down at approximately $9.80 \mathrm{~m} / \mathrm{s}^{\wedge} 2$. The experiment was left to run for the entirety of its approximately 25 parabolas of alternating $0 \mathrm{~g}$ and $2 \mathrm{~g}$ phases.

\section{Results}

The experiment was ground tested (See Graph 1) under 1g, resting normally on a lab table for a period of 15 minutes. You can see convection in the fact that the higher elevation probes (A, B, and $\mathrm{C}$ with $\mathrm{A}$ being the highest) gain temperature faster than the probes underneath the heating element. There is some 'noise' in the data that we believe was caused by static electricity on the Styrofoam that interfered with our thermocouples. 


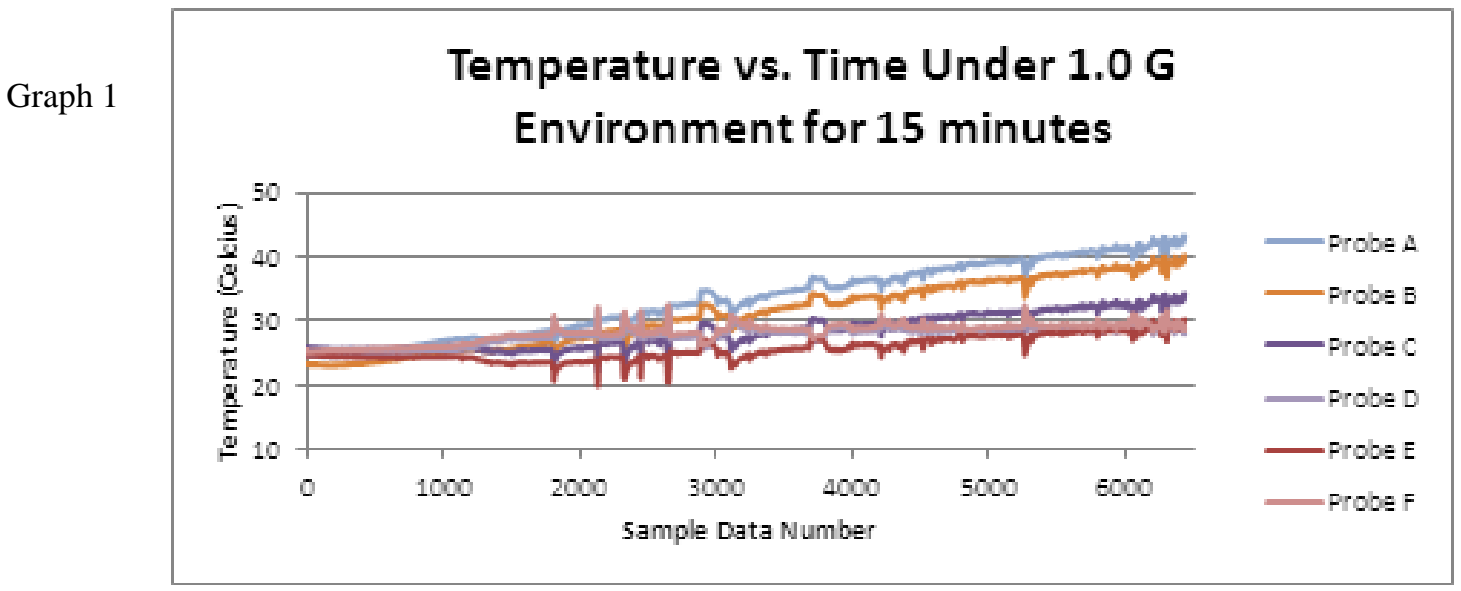

Our zero g results were disappointing due to two unforeseen events. On its first flight, there was a computer glitch that froze the taking of data. We (East Troy High School) were working with a particular team member from UW Madison who wrote and designed the program for collecting our data. He was not on that flight and the team members who were on the flight to operate our seemingly simple experiment did not know how to fix the issue. Upon landing the glitch was fixed and the experiment was ready to go, it was again ground tested, was showing good data, and was loaded with our direct contact from UW Madison for execution in midair. Upon turning on the experiment, the fuse protecting the variac blew and another was not available on the plane. The cause of the fuse blowing has not been confirmed. We believe it to be that the variac was turned too high (there was a sticker on the dial showing where it should be), the internal nichrome wire somehow touched together making it shorter and thus having a lower resistance and drawing more current, or there is was a short somewhere that we are missing. These two unfortunate events were very disappointing to both our team at ETHS and UW Madison, we feel like we let everyone down, and we believe we could have better prepared.

\section{Conclusion}

Although we were not able to collect data in a microgravity environment, we learned a valuable lesson about being prepared for anything that could go wrong when conducting experiments in space / microgravity, we gained a wealth of knowledge of how science works in the real world, and we turned some impressionable youth on to the aerospace industry. Two students are planning on starting their own Zero $G$ teams at their respective universities in the fall; Duke and UW LaCrosse. One has decided that aerospace engineering will now be her course of study when she gets to college in the fall. Two are planning on majoring in computer science and would like to intern for NASA during their college careers.

We still have options and plans for this experiment in the future:

A) Next year's incoming group of seniors is looking into the HUNCH. (High School Students Uniting with NASA to Create Hardware) If we end up going that route, we could ask and bring this fixed experiment along as a peripheral and gather data.

B) We could remedy the experiment and if UW Madison applies and is selected next year, we could send it down with them again to try and collect good data. 
If we end up sending this experiment on the plane again, we will make sure that the UW Madison team member that worked with us to develop our experiment is on the plane to assure we get good data, and we will send up an extra fuse(s) in case one burns up unexpectedly. 